\title{
Use of Mean Platelet Volume as an Initial Diagnostic Marker in Evaluation of Dengue Fever
}

\author{
Nabila Afsar*, Idrees Akhtar Afroze, Shahmeen Humaira and Zakia Abid \\ Department of Pathology, Deccan College of Medical sciences, Hyderabad, Telengana. India
}

\begin{abstract}
Background: Dengue fever is the most rapidly spreading mosquito-borne viral disease. Four main characteristic manifestations of dengue illness are (i) continuous high fever lasting 2-7 days; (ii) haemorrhagic tendency as shown by a positive tourniquet test, petechiae or epistaxis; (iii) thrombocytopoenia (platelet count $<100 \times 109 / 1$ ); and (iv) evidence of plasma leakage manifested by haemoconcentration (an increase in haematocrit $20 \%$ above average for age, sex and population), pleural effusion and ascites, etc. Recently, novel platelet indices such as MPV, PDW, and P-LCR have been investigated as prospective platelet activation markers .Platelet volume, a marker of platelet function and activity is measured as mean platelet volume (MPV) by hematology analyzer.
\end{abstract}

Method: The study was done with the aim to assess whether mean platelet volume has any diagnostic importance in the initial evaluation of dengue fever . 45 adult patients were diagnosed with Dengue NS1 antigen positivity and underwent routine complete blood counts on automated hematology analyser HORIBA Pentra ES60.

Result: A total of 76 cases ranging from 6 months to 50 years of age were reported as antigenically positive for dengue indicating acute infection, out of which 45 were adults ranging from 12 to 50 years of age. 34 were male and 11 were female. Infants and children below 12 years of age were not included in this study. 55 controls were studied which included 31 males and 24 females. The mean platelet volume was found to be significantly higher in dengue cases when compared to controls. MPV was also noted to be significantly higher in patients with platelet count below the normal biological reference range of 1.5 lakhs, when compared to dengue patients with normal platelet counts.

Conclusion: To conclude, high MPV indicates platelet activation and may be used as an initial marker to suspect dengue fever in a case of thrombocytopenia.

Keywords: Dengue Fever, MPV, Mean Platelet Volume

\section{Introduction}

Dengue fever is the most rapidly spreading mosquitoborne viral disease in the world. An estimated 50 million infections per years occur across approximately 100 countries. Incidence has increased 30 -fold with increasing geographic expansion with potential for further spread ${ }^{[1]}$ .It is transmitted mainly by Aedes aegypti mosquito and also by Aedes albopictus. All four serotypes can cause the full spectrum of disease from a subclinical infection to a mild self limiting disease, the dengue fever (DF) and a severe disease that may be fatal, the dengue haemorrhagic fever/dengue shock syndrome (DHF/ DSS). The WHO 2009 classification divides dengue fever into two groups: uncomplicated and severe, though the 1997 WHO classification is still widely used. ${ }^{[2]}$ The 1997 classification divided dengue into undifferentiated fever, dengue fever (DF), and dengue haemorrhagic fever (DHF). Four main characteristic manifestations of dengue illness are (i) continuous high fever lasting 2-7 days; (ii) haemorrhagic tendency as shown by a positive tourniquet test(Hess test or Rumpel-Leed capillary fragility test), petechiae or epistaxis; (iii) thrombocytopoenia (platelet count $<100 \times 109 / 1$ ); and (iv) evidence of plasma leakage manifested by haemoconcentration (an increase in haematocrit $20 \%$ above average for age, sex and population), pleural effusion and ascites, etc.

Recently, novel platelet indices such as MPV, PDW, and P-LCR have been investigated as prospective platelet activation markers .Platelet volume, a marker of platelet function and activity is measured as mean platelet volume (MPV) by hematology analyzers. Mean platelet volume is derived from impedence platelet size distribution curve and is highly dependent on technique of measurement, length and storage of blood sample prior to testing. In normal subjects, the MPV is inversely proportional to platelet count. MPV is decreased in megaloblastic anemia and bone marrow depression. It is increased in patients at risk of and following myocardial infarction and cerebral infarcts and also gives evidence of inherited macrothrombocytopenia. ${ }^{[3]}$

When platelet production is decreased, young platelets become bigger and more active, and MPV levels increase. 
Increased MPV indicates increased platelet diameter, which can be used as a marker of production rate and platelet activation. During activation, platelets' shapes change from biconcave discs to spherical, and a pronounced pseudopod formation occurs that leads to MPV increase during platelet activation. ${ }^{[4]}$

The study was undertaken with the aim of establishing if any relationship is seen to exist with Mean Platelet Volume in cases of dengue fever and to ascertain if there is any correlation with age and gender related difference.

\section{Materials and Methods}

A retrospective case control study of dengue cases was carried out in the Department of Pathology of Owaisi Hospital and Research Centre, Deccan College of Medical Sciences which is a tertiary care teaching hospital in Hyderabad. It was conducted during the seasonal peak of July 2016 to October 2016.

45 adult patients were diagnosed with Dengue NS1 antigen positivity and underwent routine complete blood counts on automated hematology analyser HORIBA Pentra ES60. Samples were collected in K3 EDTA vacutainers and processed within 2 hour of collection. Samples of 55 controls including 31apparently healthy non smoker adult male and 24 female controls were also collected and processed as above.

Inclusion criteria: All adult NS1 positive cases of dengue undergoing routine haematological investigation.

Exclusion criteria: Dengue negative patients and infants and children with antigen positivity were excluded to rule out any age related difference in platelet parameters.

Data was collected retrospectively from the laboratory records. Platelet count and MPV of cases of dengue fever and healthy controls were studied and analysed with the calculation of mean, median, standard deviation and range on Microsoft excel sheet and the statistical significance was established using the student $\mathrm{T}$ test to ascertain the $\mathrm{P}$ value.

\section{Result}

45 adult patients were reported as antigenically positive for dengue indicating acute infection, ranging from 12 to 50 years of age. 34 were male $(75.5 \%)$ and 11 were female (24.5\%). Infants and children below 12 years of age were not included in this study. 55 controls were studied which included 31 males(56.4\%) and 24 females(43.6\%). Table 1.

$86.7 \%$ of Dengue fever cases are young patients below 30 years of age with $44.4 \%$ cases seen in 21 to 30 years of age and $42.2 \%$ cases seen in the age group of 12 to 20 years. (Table 2)

A statistically significant increase in MPV was noted in cases of dengue in age groups of 12-20 and 21-30 years compared to the control group. The ages 31 to 50 years were analysed as a single group owing to the low number of cases and controls and no statistical significance was noted in this group probably due to the low number of cases though the mean was higher in dengue fever group.(Table 3 )

The mean platelet volume was found to be significantly higher in dengue cases (MPV $=8.84 \pm 1.05 \mathrm{fl}$ ) when compared to controls $(8.2 \pm 0.78 \mathrm{fl})$. (Table 4$)$

MPV was also noted to be significantly higher in dengue patients with thrombocytopenia (MPV=9.08 $\pm 1.08 \mathrm{fl}$ ) , when compared to dengue patients with normal platelet counts (MPV=8.1 $\pm 0.68 \mathrm{fl})$. (Table 5)

Table 1: Sex wise distribution of cases and controls.

\begin{tabular}{|c|c|c|c|c|}
\hline Gender & Cases & $\%$ & Controls & \% \\
\hline Male & 34 & 75.5 & 31 & 56.4 \\
\hline Female & 11 & 24.5 & 24 & 43.6 \\
\hline Total & $\mathbf{4 5}$ & $\mathbf{1 0 0}$ & $\mathbf{5 5}$ & 100 \\
\hline
\end{tabular}

Table 2: Age and gender wise distribution of cases and controls.

\begin{tabular}{|c|c|c|c|c|c|c|}
\hline \multirow{2}{*}{ AGE } & \multicolumn{3}{|c|}{ CASES } & \multicolumn{3}{c|}{ CONTROL } \\
\cline { 2 - 7 } & Male & Female & Total & Male & Female & Total \\
\hline $12-20$ & $15(33.3 \%)$ & $4(8.9 \%)$ & $19(42.2 \%)$ & $20(36.4 \%)$ & 24 & $44(80 \%)$ \\
\hline $21-30$ & $14(31.1 \%)$ & $6(13.3 \%)$ & $20(44.4 \%)$ & $7(12.7 \%)$ & 0 & $7(12.7 \%)$ \\
\hline $31-40$ & $1(2.2 \%)$ & 0 & $1(2.2 \%)$ & $4(7.3 \%)$ & 0 & $4(7.3 \%)$ \\
\hline $41-50$ & $4(8.9 \%)$ & $1(2.2 \%)$ & $5(11.1 \%)$ & 0 & 0 & 0 \\
\hline Total & $\mathbf{3 4}(\mathbf{7 5 . 5 \% )}$ & $\mathbf{1 1 ( 2 4 . 5 \% )}$ & $\mathbf{4 5 ( 1 0 0 \% )}$ & $\mathbf{3 1 ( 5 6 . 4 \% )}$ & $\mathbf{2 4}(\mathbf{4 3 . 6 \% )}$ & $\mathbf{5 5 ( 1 0 0 \% )}$ \\
\hline
\end{tabular}


Table 3: Age wise distribution of MPV in dengue cases compared to controls.

\begin{tabular}{|c|c|c|c|c|c|c|c|c|}
\hline CASES & \multicolumn{3}{|c|}{ MPV in CASES $(\mathrm{fl})$} & \multicolumn{2}{c|}{ CONTROL } & \multicolumn{2}{c|}{ MPV in CONTROLS(fl) } & \multirow{2}{*}{ P value } \\
\hline Age & Mean & SD & Range & Age & Mean & SD & Range & \\
\hline $12-20(n=19)$ & 8.85 & 1.10 & $7.3-10.8$ & $12-20(n=44)$ & 8.23 & 0.84 & $6.5-10.5$ & 0.04 \\
\hline $21-30(n=20)$ & 9 & 0.94 & $7.6-10.6$ & $21-30(n=7)$ & 8.29 & 0.48 & $7.7-9.1$ & 0.02 \\
\hline$>30(n=6)$ & 8.25 & 0.99 & $7-9.6$ & $>30(n=4)$ & 7.88 & 0.35 & $7.3-8.2$ & 0.46 \\
\hline
\end{tabular}

Table 4: Statistical evaluation in cases of dengue versus normal controls

\begin{tabular}{|c|c|c|c|}
\hline MPV(fl) & Cases(n=45) & Control(n=55) & T test \\
\hline Mean & 8.84 & 8.2 & \multirow{2}{*}{ P value = 0.0015 } \\
\hline Median & 8.8 & 8.2 & \\
\hline SD & 1.05 & 0.78 & \\
\hline
\end{tabular}

Table 5: MPV according to platelet count in dengue cases.

\begin{tabular}{|c|c|c|c|}
\hline MPV(fl) & $\begin{array}{c}\text { Platelet count }<150 \times 10^{3} / \\
\mathbf{m m}^{3}(\mathbf{n = 3 4 )}\end{array}$ & $\begin{array}{c}\text { Platelet count }>150 \times 10^{3} / \\
\mathbf{m m}^{3}(\mathbf{n = 1 1 )}\end{array}$ & T test \\
\hline Mean & 9.08 & 8.1 & \multirow{2}{*}{ P value $=0.001946$} \\
\hline Median & 9.15 & 8.1 & \\
\hline SD & 1.08 & 7.68 & \\
\hline
\end{tabular}

\section{Discussion}

Dengue fever was noted to be predominant in males in the present study which is consistent with study done by Martha Anker et al who found a male predominance in the reported number of incident dengue cases among persons 15 years or older in several Asian countries. ${ }^{[5]}$ Three independent studies from epidemics in India done by Agarwal et al., Ray et al., and Wali et al., also found nearly twice the number of male patients infected with dengue compared to females, M:F being 1.9:1, 1:0.57, and 2.5:1, respectively. ${ }^{[6],[7],[8],[9]}$

Dengue fever was seen to be more rampant in younger age group. Cecilia D observed that the 21-30 years age group was most affected by dengue throughout the 6 years. ${ }^{[10]}$ This was consistent with our study with $86.7 \%$ dengue fever patients being under 30 years of age.

Normal platelet size (mean platelet volume) varies between 7.5 and 10.5 fl. Some patients with severe thrombocytopenia have little or no bleeding, and show morphologically large platelets on blood smears. This information has spawned the idea that some individuals have hyperfunctional platelets that compensate for low numbers by their increased effectiveness. ${ }^{[11]}$ A literature has developed that supports the idea that young platelets, presumably those recently released from bone marrow, are larger and more dense, contain more of certain proteins(i.e. PF4), and exhibit some alteration in function as compared to smaller platelets. ${ }^{[12,13]}$ These observations may explain the findings of significant increased $\operatorname{MPV}(9.08 \mathrm{fl} \pm 1.08$ fl) in cases of dengue fever with thrombocytopenia when compared to $\operatorname{MPV}(8.1 \mathrm{fl} \pm 0.68 \mathrm{fl})$ in dengue fever cases with normal platelet count.

Basu et al suggested that DV-2 inhibits in vitro megakaryopoiesis and induces apoptotic cell death in a subpopulation of early megakaryocytic progenitors which may contribute to thrombocytopenia in dengue disease. ${ }^{[14]}$ Ghosh et al showed that DV-2 may directly interact with and activate platelets and thus may be responsible for thrombocytopenia. ${ }^{[15]}$

This study may explain the findings of the present study with MPV being significantly higher in cases of dengue fever when compared to controls due to the activation of platelets causing a rise in MPV. Budak YU discussed that when platelet production is decreased, young platelets become bigger and more active, and MPV levels increase. Increased MPV indicates increased platelet diameter, which can be used as a marker of production rate and platelet activation. During activation, platelets' shapes change from biconcave discs to spherical, and a pronounced pseudopod formation occurs that leads to MPV increase during platelet activation. ${ }^{[16]}$ This analogy also explains the high MPV noted in the present study.

However, other studies were found to show conflicting results. Prakash G. M. found no significant difference was observed in Mean between MPV at the time of minimal platelet counts and at discharge in dengue cases except in dengue fever cases. ${ }^{[17]}$ This was correlated with study done by Dewi et al ${ }^{[18]}$ and Wiwanitkit et al ${ }^{[19]}$ who suggested that MPV is not decreased in patients with dengue haemorrhagic fever and appears to be similar to that of healthy population. Bashir $\mathrm{AB}$ et al found significant 
differences in the MPV, PDW and PLT in patients with dengue infection, low platelet count, MPV, and PDW may be used as probable indicators for dengue in endemic area. Low MPV $<9$ fl and high PDW $>13 f l$ shows considerable sensitivity for dengue fever. ${ }^{[20]}$ Kritika Sharma et al showed that MPV showed no significant correlation with severity, serology \& treatment outcome, thus excluding its role in dengue cases. Mean platelet volume was not found to be an important prognostic parameter in dengue fever cases ${ }^{[4]}$.

\section{Conclusion}

Dengue fever had a higher incidence in males and in individuals younger than 30 years of age. MPV was significantly higher in patients of Dengue fever when compared to healthy controls. It was found to be significantly higher in dengue cases with low platelet count. This suggests that dengue virus causes activation of platelets leading to their subsequent increase in size and MPV. It could also be related to previous treatment history which may be stimulating new platelet formation. To conclude, high MPV indicates platelet activation and may be used as an initial marker to suspect dengue fever in a case of thrombocytopenia. However, considering the small sample size, further studies may be undertaken during future epidemics to evaluate a larger population in order to use MPV as an initial marker available in a routine hematology analyser report to avoid unnecessary serological testing

\section{Reference}

1. WHO. Dengue hemorrhagic fever: Diagnosis, treatment, prevention and control. Geneva: WHO press; 2009:3-106.

2. Dengue: Guidelines for diagnosis, treatment, prevention, and control in sub-Saharan Africa and 13 countries in South America. Geneva: World Health Organization; 2009. WHO.

3. Dacie and Lewis Practical Hematology 11th edition:47-48

4. Sharma K, Yadav A. Association of Mean Platelet Volume with Severity, Serology \& Treatment Outcome in Dengue Fever: Prognostic Utility J Clin Diagn Res. 2015; 9(11): EC01-EC03

5. Anker M, Arima Y. Male-female differences in the number of reported incident dengue fever cases in six Asian countries. Western Pacific Surveillance and Response Journal. 2011, 2(2):17-23. doi:10.5365/wpsar.2011.2.1.002

6. Guha-Sapir D, Schimmer B. Dengue fever: New paradigms for a changing epidemiology. Emerg Themes Epidemiol 2005;2:1.
7. Agarwal R, Kapoor S, Nagar R, Misra A, Tandon R, Mathur A, et al. A clinical study of the patients with dengue hemorrhagic fever during the epidemic of 1996 at Lucknow, India. Southeast Asian J Trop Med Public Health 1999;30:735-40.

8. Ray G, Kumar V, Kapoor AK, Dutta AK, Batra S. Status of antioxidants and other biochemical abnormalities in children with dengue fever. J Trop Pediatr 1999;45:4-7.

9. Wali JP, Biswas A, Handa R, Aggarwal P, Wig N, Dwivedi SN. Dengue haemorrhagic fever in adults: A prospective study of 110 cases. Trop Doct 1999;29:27-30.

10. McMillan R. Therapy for adults with refractory chronic immune thrombocytopenic purpura. Ann Intern Med 1997; 126:307-314

11. Cecilia D. Current status of dengue and chikungunya in India. WHO South-East Asia Journal of Public Health | JanMar 2014 | 3 (1) 22-27

12. Karpatkin S. Heterogeneity of human platelets. VI. Correlation of platelet function with platelet volume. Blood 1978;51:307-316

13. Wintrobe's Clinical Heamatology, 11 th edition: 632

14. Basu A, Jain P, Gangodkar S, Shetty S, Ghosh K. Dengue 2 virus inhibits in vitro megakaryocytic colony formation and induce apoptosis in thrombopoietin-inducible megakaryocytic differentiation from cord blood CD34+ cells. FEMS Immunol Med Microbiol. 2008;53:46-51.

15. Ghosh K, Gangodkar S, Jain P, Shetty S, Ramjee S, Poddar $\mathrm{P}$, et al. Imaging the interaction between dengue 2 virus and human blood platelets using atomic force and electron microscopy. J Electron Microsc. 2008;57:113-8.

16. Budak YU, Polat M, Huysal K. The use of platelet indices, plateletcrit, mean platelet volume and platelet distribution width in emergency non-traumatic abdominal surgery: a systematic review. Biochemia Medica. 2016;26(2):178-193.

17. Prakash GM, Anikethana G V. Use of mean platelet volume and platelet distribution width in predicting trend in platelet count and bleeding risks in patients of dengue fever. Int $\mathrm{J}$ Adv Med 2016;3:611-3.

18. Dewi YP. Mean Platelet Volume (MPV): Potential Predictor of Disease Severity in Dengue infection. In proceeding of: International Dengue Symposium. 2013 conference paper.

19. Wiwanitkit V. Mean platelet volume in the patients with dengue hemorrhagic fever. Platelets. 2004;15(3):185.

20. Bashir AB, Saeed OK, Mohammed BA, Ageep AK. Role of Platelet Indices in Patients with Dengue Infection in Red Sea State, Sudan. International Journal of Science and Research 2015;4:1573-1576

*Corresponding author:

Dr. Nabila Afsar, 5-5-203/3, Patel Nagar, Nampally, Hyderabad, 500001, Telengana, India

Phone: +91 9848020386

Email: nabila_dr@yahoo.com

Financial or other Competing Interests: None.

Date of Submission : 14.03.2017

Date of Acceptance : 11.04.2017

Date of Publication : 04.07.2017 